Formation of various metal nanostructures with thermal annealing to control the effective coupling energy between a surface plasmon and an InGaN/GaN quantum well

This article has been downloaded from IOPscience. Please scroll down to see the full text article.

2007 Nanotechnology 18265402

(http://iopscience.iop.org/0957-4484/18/26/265402)

The Table of Contents and more related content is available

Download details:

IP Address: 140.112.113.225

The article was downloaded on 20/02/2009 at 05:42

Please note that terms and conditions apply. 


\title{
Formation of various metal nanostructures with thermal annealing to control the effective coupling energy between a surface plasmon and an InGaN/GaN quantum well
}

\author{
Dong-Ming Yeh, Cheng-Yen Chen, Yen-Cheng Lu, \\ Chi-Feng Huang and C C Yang \\ Graduate Institute of Electro-Optical Engineering and Department of Electrical Engineering, \\ National Taiwan University, 1, Roosevelt Road, Section 4, Taipei, Taiwan, Republic of China \\ E-mail: ccy@cc.ee.ntu.edu.tw
}

Received 7 March 2007, in final form 24 April 2007

Published 5 June 2007

Online at stacks.iop.org/Nano/18/265402

\begin{abstract}
We demonstrate the variations of the photoluminescence (PL) spectral peak position and intensity through the surface plasmon (SP) coupling with an InGaN/GaN quantum well (QW) by forming Ag nanostructures of different scale sizes on the QW structure with thermal annealing. By transferring an $\mathrm{Ag}$ thin film into a nanoisland structure, we can not only enhance the PL intensity, but also adjust the SP dispersion relation and hence red-shift the effective QW emission wavelength. Such an emission spectrum control can be realized by initially coating Ag films of different thicknesses. Although the screening process of the quantum-confined Stark effect, which can result in PL spectrum blue-shift and intensity enhancement, also contributes to the variations of the emission behaviour, it is found that the SP-QW coupling process dominates in the observed phenomena.
\end{abstract}

(Some figures in this article are in colour only in the electronic version)

\section{Introduction}

InGaN/GaN quantum well (QW) structures have been widely used for manufacturing UV-blue-green light-emitting diodes (LEDs). Such a QW structure was also used for the fabrication of orange-red LEDs [1, 2]. Just like other light-emitting devices, the InGaN/GaN QW-based LEDs suffer from low external quantum efficiency unless certain light extraction mechanisms are used. Meanwhile, as the indium content increases for emitting longer-wavelength visible light, the internal quantum efficiency drops tremendously even when special growth techniques are used [1,3]. Therefore, certain means for enhancing light emission and light extraction are important for developing all-InGaN colour or white-light LEDs.

Recently, photoluminescence (PL) enhancement of an InGaN/GaN QW through the coupling of the dipoles in the
QW with the surface plasmons (SPs) generated at the interface between the semiconductor and an Ag thin film coated on the top was reported $[4,5]$. In such a coupling process, the dipole energy in the QW is transferred into one or more SP modes through the coverage of the SP evanescent field over the QW. Then, the SP modes can radiate if the phase matching condition between SP and photon is reached. The $S P$ radiation represents another emission channel besides the direct dipole radiation. Depending on several factors, including the non-radiative recombination rate of the dipole, the loss rate of the coupled SP mode, the availability of the phasematching condition for SP radiation, and the SP coupling efficiency, the PL can be enhanced or suppressed [6]. It is believed that the SP-QW coupling process should be useful for enhancing the overall quantum efficiency of an LED, particularly in those light emission structures of low internal 
quantum efficiency. The use of the SP-QW coupling in an LED also takes advantage of the fact that a metal layer is always needed for Ohmic contact or current spreading in an LED. The metal layer can also serve to generate SPs.

In the SP-QW coupling process, one of the key factors for effective light emission enhancement is the phase-matching condition between SP and photon [7]. The phase-matching condition is controlled by the dispersion relation of the SP mode involved in the SP-QW coupling process. The SP dispersion relation is determined by the dielectric property of the metal as well as its nanostructure [8]. Therefore, once a metal is selected for an LED emission wavelength range, the metal nanostructure becomes the key factor for controlling the SP dispersion relation. A well defined periodical metal nanostructure or grating, which can be fabricated with electron-beam lithography or nanoimprint and certain dry etching techniques, is usually considered to effectively adjust the dispersion relation. However, the use of the electron-beam lithography or nanoimprint can be too expensive or complicated in LED fabrication. Simple methods for metal nanostructure formation are needed to make the application of SP-QW coupling to LED fabrication practical. A random nanostructure can be decomposed into various gratings of different spatial-frequency or wavenumber components. Certain components can be used for momentum matching between SPs and photons. A finer nanostructure can provide the grating components of more effective momentum matching.

In this paper, we demonstrate the use of the thermal annealing technique for forming metal nanostructures on the top of an InGaN/GaN QW to adjust the effective emission wavelength through the SP-QW coupling $[9,10]$. It is found that under the same annealing conditions, different coated $\mathrm{Ag}$ thicknesses lead to the nanostructures of different scale sizes, which result in different PL spectral peaks. Timeresolved PL (TRPL) properties are measured to help the interpretation. In section 2 of this paper, the sample structures and the experimental procedures are presented. The metal nanostructures under different fabrication conditions are shown in section 3. The PL and TRPL behaviours of various samples of different metal nanostructures are reported in section 4 . Discussions and conclusions are given in section 5.

\section{Sample preparation and experimental procedures}

The InGaN/GaN QW epitaxial structure was grown with metallorganic chemical vapour deposition. It consists of an InGaN QW layer (grown at $710^{\circ} \mathrm{C}$ ) of $3 \mathrm{~nm}$ in thickness sandwiched by a top $\mathrm{GaN}$ cap layer (grown at $850^{\circ} \mathrm{C}$ ) of $26 \mathrm{~nm}$ in thickness and the lower GaN cladding layer (grown at $1070{ }^{\circ} \mathrm{C}$ ) of $2 \mu \mathrm{m}$ in thickness. The semiconductor surface root-mean-square (rms) roughness is about $0.5 \mathrm{~nm}$. Based on the temperature-dependent PL measurement, the internal quantum efficiency was estimated to be $20 \%$. Three samples were prepared by coating Ag thin films of different thicknesses at 5, 10 and $15 \mathrm{~nm}$ for comparison. The $\mathrm{Ag}$ thin films were deposited at room temperature with an electron-gun coater. The thermal annealing was performed under the conditions of $200^{\circ} \mathrm{C}$ for $30 \mathrm{~min}$. Such low-temperature annealing conditions do not affect the semiconductor nanostructure. In this research,
$\mathrm{Ag}$ was chosen for generating SPs because the SP resonance energy of bulk Ag corresponds to $\sim 440 \mathrm{~nm}$ in wavelength $(\sim 2.82 \mathrm{eV})$, which is slightly shorter than the PL spectral peak of the grown QW sample at $447 \mathrm{~nm}$.

Both the PL and TRPL measurements were excited by the second harmonic of a femtosecond Ti:sapphire laser at $780 \mathrm{~nm}$. The excitation and PL signal collection were preformed from the polished backside of the sample. The PL spectra were carefully measured such that the spectral intensities of different samples can be compared. The TRPL profiles were recorded with a streak camera of $50 \mathrm{ps}$ in temporal resolution.

\section{Metal nanostructures}

Figures 1(a) and (b) show the scanning electron microscopy (SEM) images on the metal surfaces of the $5 \mathrm{~nm}$ sample before annealing (a) and after annealing (b), respectively. Parts (c) and (d) of figure 1 show the line-scan profiles in an atomic-force microscopy (AFM) measurement of the same sample before and after annealing, respectively. One can see that before annealing of this sample Ag nanocrystals with the scales of 30$35 \mathrm{~nm}$ in width and $4 \mathrm{~nm}$ in height (4.3 $\mathrm{nm}$ in rms roughness) are formed. After thermal annealing, the Ag nanocrystals become more aggregated to form nanodots of $80-100 \mathrm{~nm}$ in width and $15-20 \mathrm{~nm}$ in height (16 $\mathrm{nm}$ in rms roughness).

Figures 2(a)-(d) show the SEM images on the metal surfaces of the $10 \mathrm{~nm}$ sample before annealing (a) and after annealing (b), and the AFM line-scan profiles before (c) and after (d) annealing, respectively. In this sample, although a relatively flatter metal surface $(35-40 \mathrm{~nm}$ in scale size and $5.1 \mathrm{~nm}$ in rms roughness) similar to that of the $5 \mathrm{~nm}$ sample can be observed before thermal annealing, a pattern of irregular wired islands can be seen after thermal annealing. The width and height of the wired island range from 100 to $200 \mathrm{~nm}$ and from 20 to $40 \mathrm{~nm}$ (with $27 \mathrm{~nm}$ in rms roughness), respectively. Then, figures 3(a)-(d) show the SEM images on the metal surfaces of the $15 \mathrm{~nm}$ sample before annealing (a) and after annealing (b), and the AFM line-scan profiles before (c) and after (d) annealing, respectively. Before thermal annealing, the Ag pattern of the $15 \mathrm{~nm}$ sample is similar to that of the $10 \mathrm{~nm}$ sample. After thermal annealing, the separated wired island patterns of the two thicker Ag-film samples are also similar. However, the width and height of the wired island in the $15 \mathrm{~nm}$ sample become larger, ranging from 200 to $300 \mathrm{~nm}$ and from 30 to $60 \mathrm{~nm}$ (with $41 \mathrm{~nm}$ in rms roughness), respectively. Bare $\mathrm{GaN}$ can be seen between these islands.

\section{Photoluminescence and time-resolved photoluminescence measurements}

In figure 4, we compare the PL spectra of the bare-QW, coated, and annealed samples of $10 \mathrm{~nm} \mathrm{Ag}$ coating. One can see that with the $10 \mathrm{~nm} \mathrm{Ag} \mathrm{coating,} \mathrm{although} \mathrm{the} \mathrm{PL} \mathrm{spectral} \mathrm{peak}$ is enhanced and blue-shifted (from 447 to $440 \mathrm{~nm}$ ) relative to those of the bare-QW case, the integrated PL intensity is not significantly changed. After thermal annealing, the PL spectral peak red-shifts from the coated case of $440 \mathrm{~nm}$ to $445 \mathrm{~nm}$. Both the PL spectral peak intensity and the integrated intensity are significantly enhanced after thermal annealing. In figure 5, three TRPL profiles (with the log scale in the 
(a)

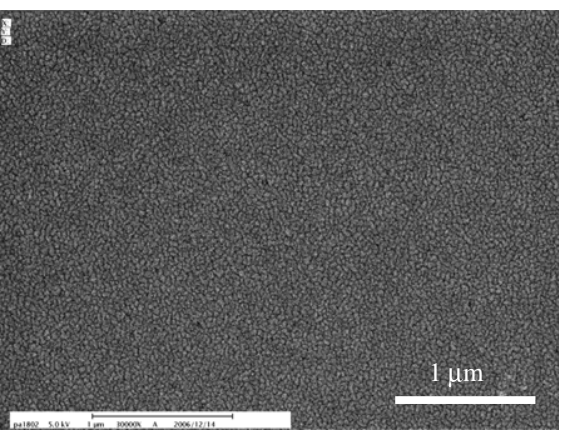

(b)

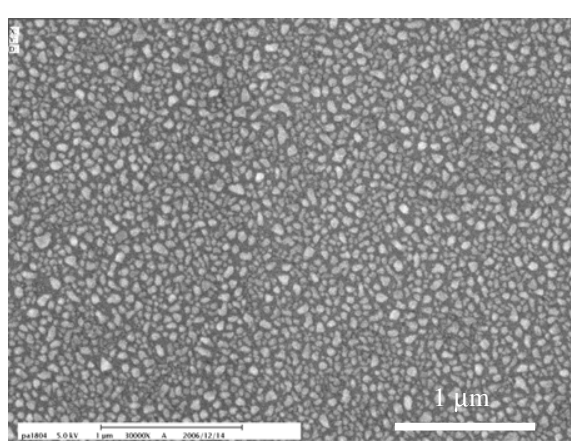

(c) $\mathrm{nm}$

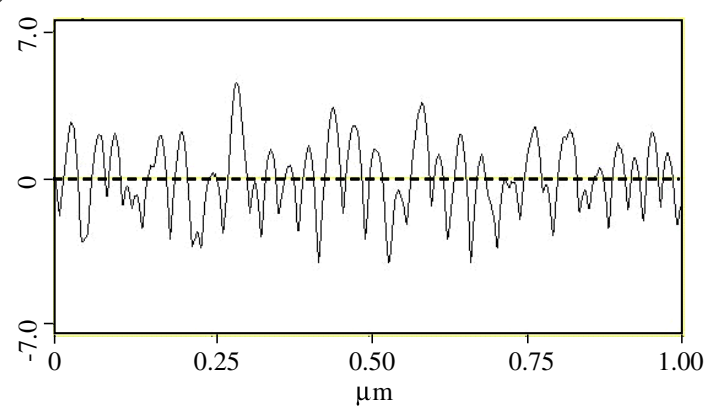

(d) $\mathrm{nm}$

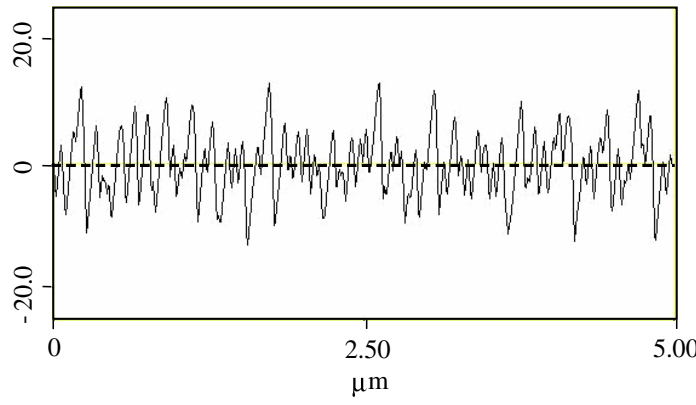

Figure 1. (a) An SEM image of the $5 \mathrm{~nm}$ sample before annealing; (b) an SEM image of the $5 \mathrm{~nm}$ sample after annealing; (c) an AFM line-scan profile of the $5 \mathrm{~nm}$ sample before annealing; (d) an AFM line-scan profile of the $5 \mathrm{~nm}$ sample after annealing.

ordinate) are shown for the three cases of figure 4. After coating and annealing, the PL decay times are reduced. At least two decay components can be observed. After coating the $10 \mathrm{~nm} \mathrm{Ag} \mathrm{film,} \mathrm{the} \mathrm{PL} \mathrm{decay} \mathrm{time} \mathrm{is} \mathrm{reduced} \mathrm{from} 7.19 \mathrm{~ns}$ to 1.06 (the faster early-stage decay) and $1.81 \mathrm{~ns}$ (the slower later-stage decay). However, after thermal annealing, the two decay times are raised to 2.80 and $4.76 \mathrm{~ns}$. In table 1 , we summarize the early- and later-stage PL decay times of the total (a)

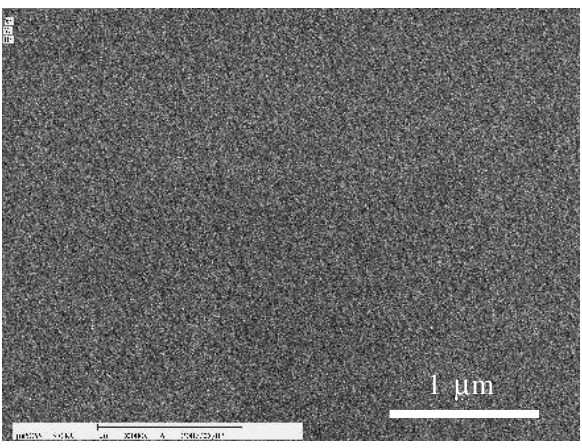

(b)

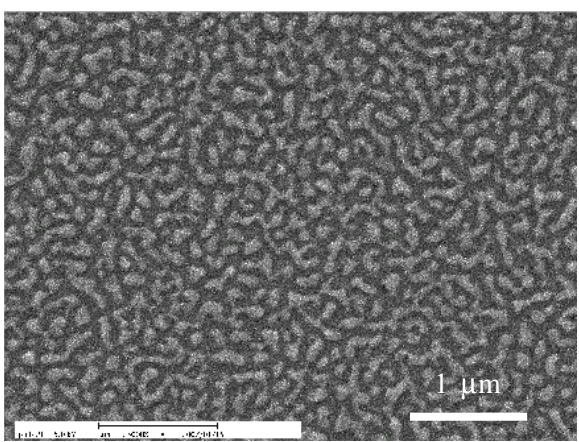

(c)

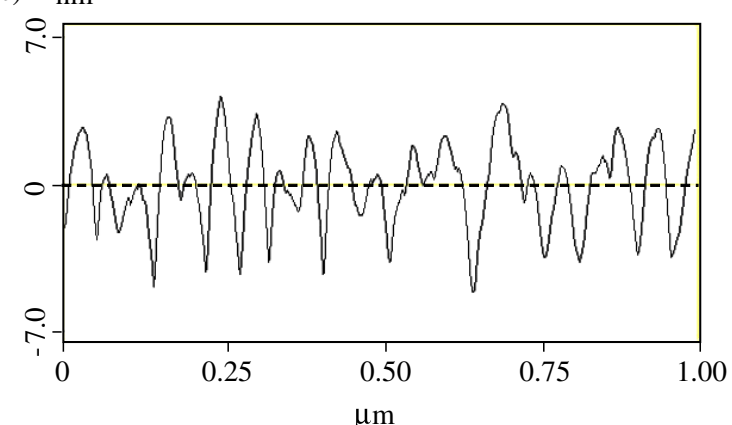

(d) $\mathrm{nm}$

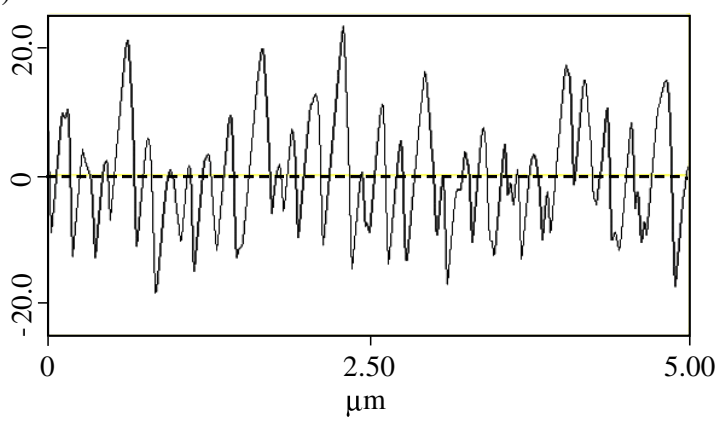

Figure 2. (a) An SEM image of the $10 \mathrm{~nm}$ sample before annealing; (b) an SEM image of the $10 \mathrm{~nm}$ sample after annealing; (c) an AFM line-scan profile of the $10 \mathrm{~nm}$ sample before annealing; (d) an AFM line-scan profile of the $10 \mathrm{~nm}$ sample after annealing.

of seven samples. The multi-stage decay is due to the carrier density dependent carrier relaxation process and a mixture of the dipole and SP radiations. Besides the SP-QW coupling, the enhanced screening process of the quantum-confined Stark effect (QCSE) due to the multi-excitation of PL (enhanced reflection at the metal-GaN interface) can also be used to interpret the enhanced and blue-shifted PL spectral peak of 
(a)

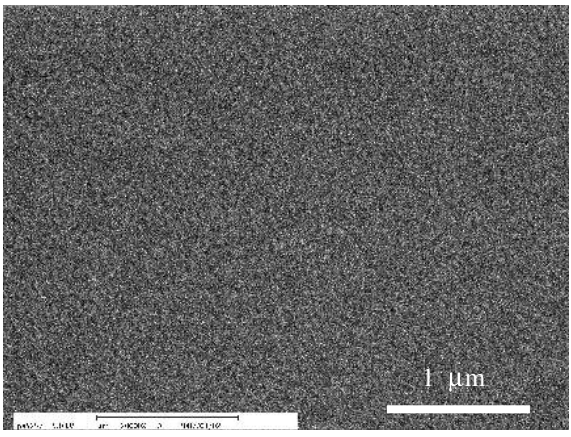

(b)

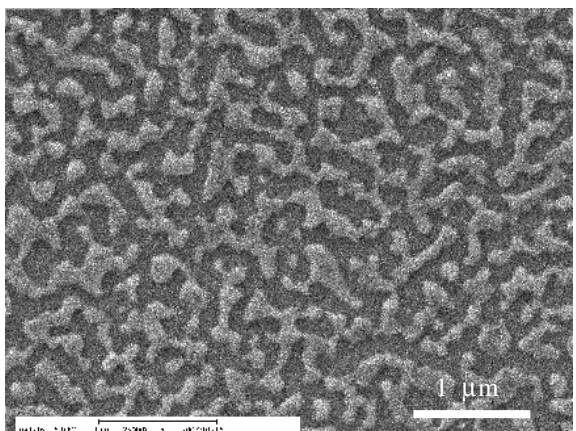

(c)

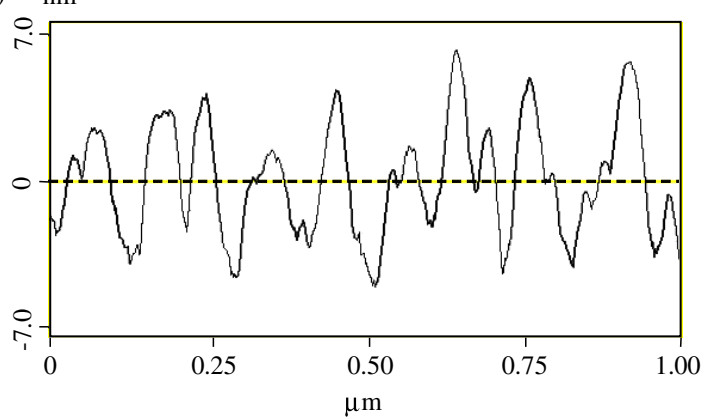

(d) $\mathrm{nm}$

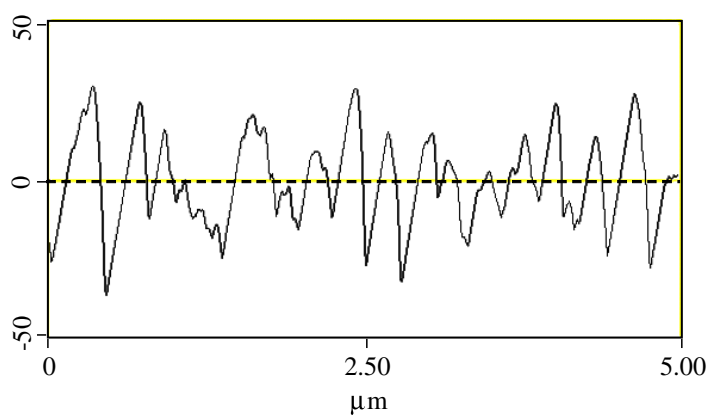

Figure 3. (a) An SEM image of the $15 \mathrm{~nm}$ sample before annealing; (b) an SEM image of the $15 \mathrm{~nm}$ sample after annealing; (c) an AFM line-scan profile of the $15 \mathrm{~nm}$ sample before annealing; (d) an AFM line-scan profile of the $15 \mathrm{~nm}$ sample after annealing.

the coated sample in figure 4 [11]. However, in the annealed sample, the SP-QW coupling process should play an important role in the emission behaviour. If the QCSE screening effect dominated, since the PL spectral peak is red-shifted from 440 to $445 \mathrm{~nm}$ after thermal annealing, we would expect that the QCSE screening effect would be reduced after annealing. In this situation, the PL intensity of the annealed sample must

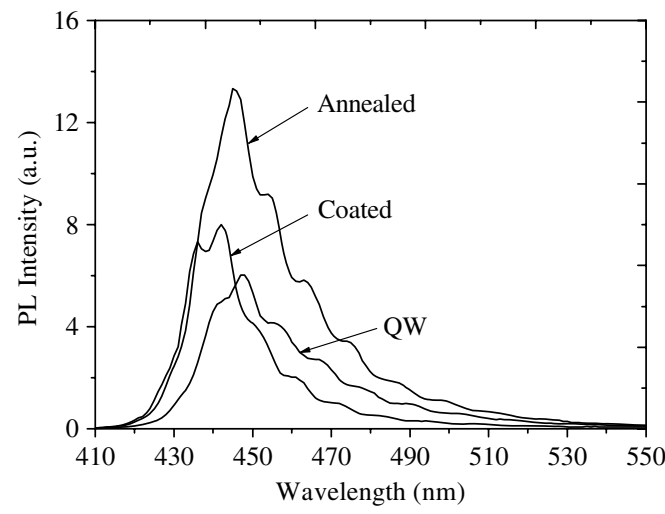

Figure 4. PL spectra of the bare-QW, coated, and annealed samples with $10 \mathrm{~nm}$ Ag coating.

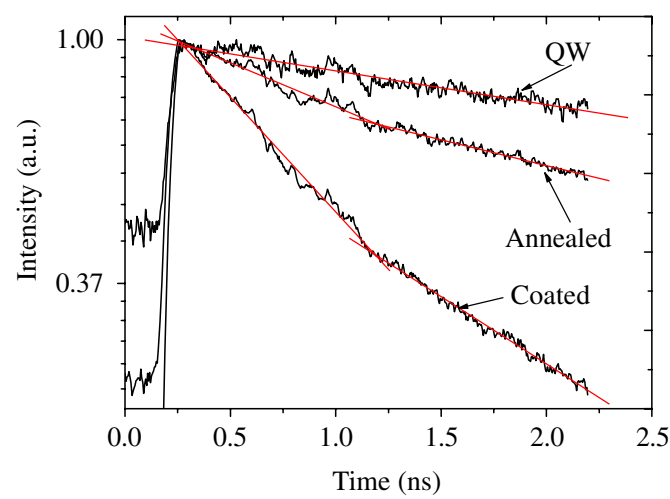

Figure 5. Time-resolved PL decay profiles of the three cases in figure 4.

Table 1. Early- and later-stage PL decay times of the seven samples.

\begin{tabular}{lll}
\hline Sample & $\begin{array}{l}\text { Early-stage PL } \\
\text { decay time (ns) }\end{array}$ & $\begin{array}{l}\text { Later-stage PL } \\
\text { decay time (ns) }\end{array}$ \\
\hline Bare-QW & 7.19 & - \\
Coated-5 nm & 3.71 & 6.01 \\
Coated-10 nm & 1.06 & 1.81 \\
Coated-15 nm & 1.00 & 1.68 \\
Annealed-5 nm & 3.09 & 4.75 \\
Annealed-10 nm & 2.80 & 4.76 \\
Annealed-15 nm & 1.88 & 3.84 \\
\hline
\end{tabular}

be weaker than that of the coated sample, that contradicts the result in figure 4. Hence, it is believed that the SP-QW coupling process plays an important role in both coated and annealed samples, particularly in the annealed sample. The faster PL decay process in the coated sample can be attributed to the stronger $\mathrm{SP}-\mathrm{QW}$ coupling. It is noted that stronger SPQW coupling does not guarantee higher PL intensity because $\mathrm{SP}$ radiation requires a phase-matching condition between the SP and the photon. Nevertheless, we cannot exclude the possibility of the stronger QCSE screening effect in the coated sample, when compared with the annealed sample.

Figure 6 shows the PL spectra of the bare QW and the three samples of different $\mathrm{Ag}$ coating thicknesses before thermal annealing. One can see that the PL spectral peaks of the 10 and $15 \mathrm{~nm}$ samples are significantly blue-shifted 


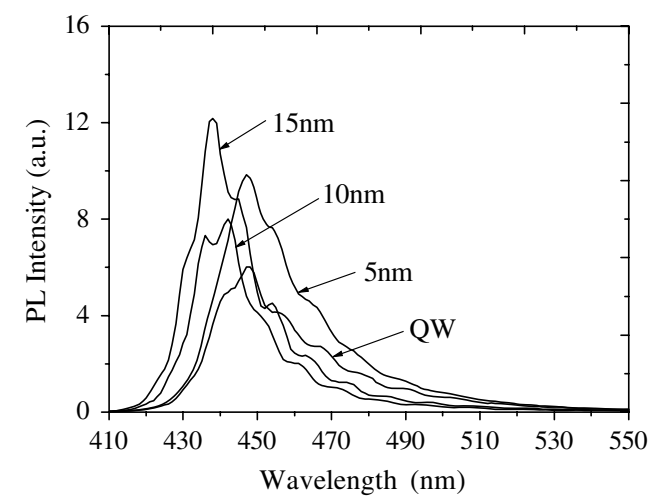

Figure 6. PL spectra of the bare QW and the three samples of different Ag coating thicknesses before thermal annealing.

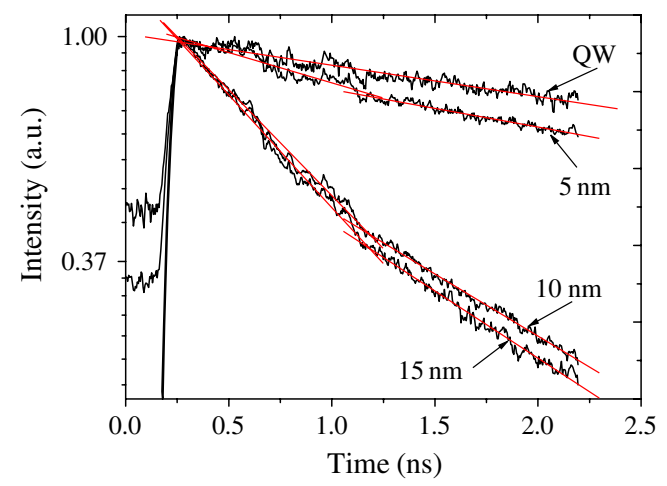

Figure 7. Time-resolved PL decay profiles of the four cases in figure 6.

from 447 to $440 \mathrm{~nm}$ and $439 \mathrm{~nm}$, respectively. However, that of the $5 \mathrm{~nm}$ sample is basically not shifted. Because the enhanced reflection with $5 \mathrm{~nm}$ coating is negligibly small and hence the enhanced QCSE screening effect can be ignored in this situation, the PL enhancement here must be attributed to the SP-QW coupling. However, because of the inherent nanostructure nature of the $5 \mathrm{~nm}$ coating (see figures 1(a) and (c)), the phase-matching condition between $\mathrm{SP}$ and photon may be more easily reached for the photons of lower energies. Such an SP-QW coupling behaviour can be understood by observing the significant PL enhancement on the long-wavelength side of the $5 \mathrm{~nm}$ sample in figure 6 . The SP-QW coupling process in this sample also results in the reduction of the PL decay time, as shown in figure 7 . Further reductions in PL decay time of the 10 and $15 \mathrm{~nm}$ samples are attributed to the mixed effect of the SP-QW coupling and the QCSE screening process.

Figure 8 shows the counterparts of figure 6 after thermal annealing. After thermal annealing, the formation of $\mathrm{Ag}$ islands changes the dispersion curves of the SPs involved in the SP-QW coupling process. Hence, the PL spectral peaks of the 5, 10, and $15 \mathrm{~nm}$ samples are red-shifted to 449, 445, and $443 \mathrm{~nm}$, respectively. Among these three samples, the SP-QW coupling can be the strongest in the $15 \mathrm{~nm}$ sample. Therefore, its PL intensity is the strongest and its PL decay times are the shortest, followed by the $10 \mathrm{~nm}$ and then the $5 \mathrm{~nm}$ samples, as shown in figure 9 .

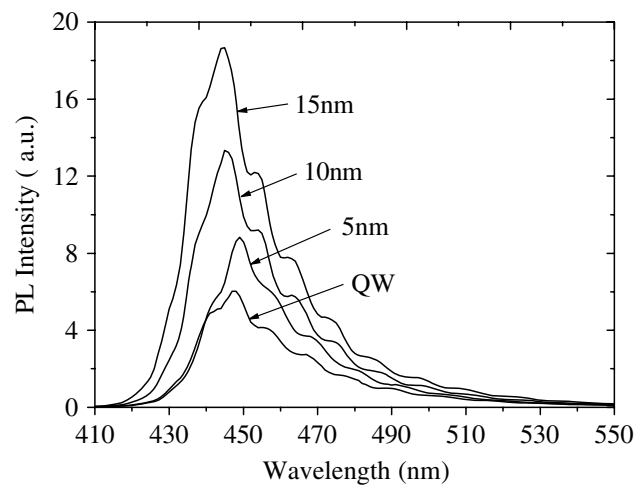

Figure 8. PL spectra of the bare QW and the three samples of different Ag coating thicknesses after thermal annealing.

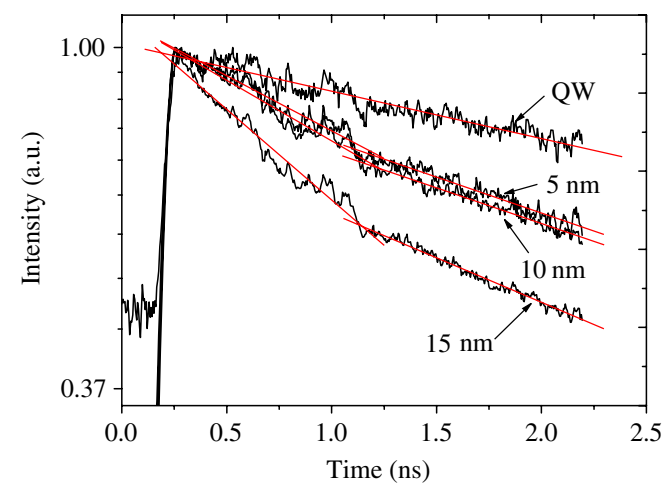

Figure 9. Time-resolved PL decay profiles of the four cases in figure 8 .

\section{Discussions and conclusions}

The PL spectral peak wavelengths and the PL spectral centreof-mass (COM) wavelengths of the total of seven cases are summarized in the second and fifth columns, respectively, of table 2. The shifts of the peak and the COM wavelengths of the three Ag-coated samples, including the cases before and after annealing, with respect to those of the bare-QW sample are listed in the third and sixth columns, respectively, of the same table. Then, the shifts of the peak and the $\mathrm{COM}$ wavelengths of the three annealed samples with respect to those of the individual as-coated samples are tabulated in the fourth and seventh columns, respectively, of this table. The plus and minus signs before the numbers of wavelength variations correspond to the results of red- and blue-shifts, respectively.

As mentioned earlier, the significant red-shift, either in PL peak energy or in COM energy, in the annealed sample of $5 \mathrm{~nm} \mathrm{Ag}$ coating implies that the SP-QW coupling effect dominates over the QCSE screening process by adding the layer of the annealed Ag nanostructure. The QCSE screening process always generates a blue-shift; however, because of the formation of the Ag nanostructure, the SP-QW coupling can significantly enhance the PL spectral portion on the low-energy side due to the favoured phase-matching condition between the SP and the photon. On the other hand, the quite large blue-shifts, in both PL peak energy and COM energy, after 
Table 2. The PL spectral peak wavelength, the PL spectral centre-of-mass wavelength, and the relative shifts. The plus and minus signs for the wavelength variations represent the red- and blue-shifts, respectively.

\begin{tabular}{|c|c|c|c|c|c|c|}
\hline \multirow[b]{2}{*}{ Sample } & \multicolumn{3}{|c|}{ PL spectral peak wavelength } & \multicolumn{3}{|c|}{ PL spectral centre-of-mass wavelength } \\
\hline & $\begin{array}{l}\text { Spectral } \\
\text { position } \\
(\mathrm{nm})\end{array}$ & $\begin{array}{l}\text { Shift from } \\
\text { the bare-QW } \\
\text { sample }(\mathrm{nm})\end{array}$ & $\begin{array}{l}\text { Shift from } \\
\text { the coated } \\
\text { sample }(\mathrm{nm})\end{array}$ & $\begin{array}{l}\text { Spectral } \\
\text { position } \\
(\mathrm{nm})\end{array}$ & $\begin{array}{l}\text { Shift from } \\
\text { the bare-QW } \\
\text { sample }(\mathrm{nm})\end{array}$ & $\begin{array}{l}\text { Shift from } \\
\text { the coated } \\
\text { sample }(\mathrm{nm})\end{array}$ \\
\hline Bare-QW & 447 & & & 458.95 & & \\
\hline Coated-5 nm & 447 & 0 & & 458.24 & -0.71 & \\
\hline Coated-10 nm & 440.01 & -6.99 & & 447.69 & -11.26 & \\
\hline Coated-15 nm & 439 & -8 & & 446.78 & -12.17 & \\
\hline Annealed $-5 \mathrm{~nm}$ & 449.02 & +2.02 & +2.02 & 459.89 & +0.94 & +1.65 \\
\hline Annealed-10 nm & 445 & -2 & +4.99 & 456 & -2.95 & +8.31 \\
\hline Annealed-15 nm & 443.02 & -3.98 & +4.02 & 454.16 & -4.79 & +7.38 \\
\hline
\end{tabular}

the coatings of the 10 or $15 \mathrm{~nm} \mathrm{Ag} \mathrm{films} \mathrm{are} \mathrm{attributed} \mathrm{to} \mathrm{the}$ enhanced SP-QW coupling and possibly the enhanced QCSE screening process. Then, after thermal annealing, because the scale sizes of the Ag nanostructures increase in all cases, the phase-matching conditions between the SP and the photon are expected to move into a range of smaller wavenumber of the SP. Hence, all the spectral features show significant redshifts. It is noted that the red-shift of either spectral feature does not monotonically follow the increased extent of the scale size of the Ag nanostructure during annealing. Although the $\mathrm{Ag}$ island widths in the annealed $15 \mathrm{~nm}$ sample are more enlarged than those of the annealed $10 \mathrm{~nm}$ sample, the redshift range of the former is smaller than that of the latter. This result can be attributed to the increased reflection at the metal/semiconductor interface such that the QCSE screening effect is somewhat enhanced to partially cancel the red-shift. However, because the Ag nanostructures are quite complicated, this issue deserves further investigation.

The other interesting observation in comparing the third and sixth columns of table 2 is that the COM energy is always more blue-shifted than the PL peak energy. The COM energy and the peak energy are related to two kinds of spectral shape variation: shift and tilt. A spectral shift can normally lead to about the same changes of the PL spectral peak and COM energies. However, a spectral tilt does not guarantee a significant change of the COM energy. In physics, the screening of QCSE is expected to generate a clear blueshift of the PL spectral peak due to the broadening of the effective bandgap. However, when a weak SP-QW coupling process occurs, the PL spectrum may simply tilt due to the energy-dependent SP density-of-state distribution. Therefore, the significantly larger blue-shifts in the COM energy, when compared with those in PL peak energy, imply that the factor of $\mathrm{SP}-\mathrm{QW}$ coupling should dominate in the process of generating those blue-shifts of the PL features. The same argument can be applied to the red-shift observations in the fourth and seventh columns of table 2 because the reflection at the metal-semiconductor interface cannot be significantly increased during thermal annealing. This reflection level determines the strength of the screening effect of QCSE. The SP density of states decreases as the point of the phasematching between the SP and the photon moves toward a lower energy, i.e. farther away from the SP resonance energy. Hence, after thermal annealing, the SP-QW coupling becomes weaker as indicated by the increases of the PL decay times after thermally annealing the 10 and $15 \mathrm{~nm}$ samples (compare figures 7 and 9). Nevertheless, after thermal annealing the PL intensities are enhanced because of the better phase matching between the SP and the photon. On the other hand, in the sample of $5 \mathrm{~nm} \mathrm{Ag}$ coating, after thermal annealing the PL decay times become shorter. In this situation, the SP-QW coupling strength may be enhanced. However, the PL intensity is essentially unchanged.

In summary, we have demonstrated the variations of the PL spectral peak position and intensity through the SP-QW coupling by forming Ag nanostructures of different scale sizes with thermal annealing on an InGaN/GaN QW structure. By transferring an $\mathrm{Ag}$ thin film into a nanoisland structure, we could not only enhance the PL intensity, but also change the SP dispersion relation and hence red-shift the effective QW emission wavelength. Such an emission spectrum control could be realized by coating Ag films of different thicknesses.

\section{Acknowledgments}

This research was supported by the National Science Council, The Republic of China, under grants NSC 95-2120-M-002-012 and NSC 95-2221-E-002-287, and by US Air Force Scientific Research Office under contract AOARD-06-4052. Support from the Centre of Nano Science and Technology, National Taiwan University, is also acknowledged.

\section{References}

[1] Huang C F, Tang T Y, Huang J J, Shiao W Y, Yang C C, Hsu C W and Chen L C 2006 Appl. Phys. Lett. 89051913

[2] Chen H S, Lu C F, Yeh D M, Huang C F, Huang J J and Yang C C 2006 IEEE Photon. Technol. Lett. 182269

[3] Kikuchi A, Kawai M, Tada M and Kishino K 2004 Japan. J. Appl. Phys. 43 L1524

[4] Okamoto K, Niki I, Shvartser A, Narukawa Y, Mukai T and Scherer A 2004 Nat. Mater. 3601

[5] Okamoto K, Niki I, Scherer A, Narukawa Y, Mukai T and Kawakami Y 2005 Appl. Phys. Lett. 87071102

[6] Chen C Y, Yeh D M, Lu Y C and Yang C C 2006 Appl. Phys. Lett. 89203113

[7] Neogi A, Lee C-W, Everitt H O, Kuroda T, Tackeuchi A and Yablonvitch E 2002 Phys. Rev. B 66153305

[8] Barnes W L, Dereux A and Ebbesen T W 2003 Nature 424824

[9] Pillai S, Catchpole K R, Trupke T, Zhang G, Zhao J and Green M A 2006 Appl. Phys. Lett. 88161102

[10] Choulis S A, Mathai M K and Choong V-E 2006 Appl. Phys. Lett. 88213503

[11] Choi C K, Kwon Y H, Little B D, Gainer G H, Song J J, Chang Y C, Keller S, Mishra U K and DenBaars S P 2001 Phys. Rev. B 64245339 